\title{
Risk factors and outcomes among children admitted to hospital with pandemic H1N1 influenza
}

\author{
Sean O'Riordan MB BChir PhD, Michelle Barton MBBS DM, Yvonne Yau MD, \\ Stanley E. Read MD PhD, Upton Allen MBBS MSc, Dat Tran MD MSc \\ Previously published at www.cmaj.ca
}

\section{ABSTRACT}

Background: Limited data are available on disease characteristics and outcomes of children with 2009 pandemic influenza $A(H 1 N 1)$ virus infection (pandemic H1N1 influenza) who have required hospital admission.

Methods: We reviewed the charts of 58 children with pandemic H1N1 influenza admitted to a large pediatric hospital in Ontario, Canada, between May 8 and July 22, 2009. We compared risk factors, severity indicators and outcomes of these children with those of 200 children admitted with seasonal influenza A during the previous 5 years (2004/05 to 2008/09).

Results: Children with pandemic H1N1 influenza were significantly older than those with seasonal influenza (median age 6.4 years v. 3.3 years). Forty-six (79\%) of the children with pandemic H1N1 influenza had underlying medical conditions; of the other 12 who were previously healthy, $42 \%$ were under 2 years of age. Children admitted with pandemic H1N1 influenza were significantly more likely to have asthma than those with seasonal influenza ( $22 \%$ v. $6 \%$ ). Two children had poorly controlled asthma, and 6 used inhaled medications only intermittently. The median length of stay in hospital was 4 days in both groups of children. Similar proportions of children required admission to the intensive care unit $(21 \%$ of those with pandemic H1N1 influenza and $14 \%$ of those with seasonal influenza) and mechanical ventilation (12\% and $10 \%$ respectively). None of the children admitted with pandemic H1N1 influenza died, as compared with $1(0.4 \%)$ of those admitted with seasonal influenza.

Interpretation: Pandemic H1N1 influenza did not appear to cause more severe disease than seasonal influenza A. Asthma appears to be a significant risk factor for severe disease, with no clear relation to severity of asthma. This finding should influence strategies for vaccination and pre-emptive antiviral therapy.

I nfluenza causes significant morbidity and mortality in childhood. ${ }^{1}$ Infants, young children and people 65 years of age and older account for the highest rates of influenza-related hospital admission. ${ }^{2}$ Earlier case series of 2009 pandemic influenza $\mathrm{A}(\mathrm{H} 1 \mathrm{~N} 1)$ virus infection (pandemic H1N1 influenza) reported small numbers of children ${ }^{3,4}$ or did not present data on children separately. ${ }^{5}$ A recently published series that included 122 children confirmed typical influenzalike presentation, reported a high prevalence of underlying medical conditions $(60 \%$, including asthma in $29 \%)$ and described the need for intensive care in $20 \%$ and mechanical ventilation in $10 \% .{ }^{6} \mathrm{~A}$ previous comparison of children with pandemic H1N1 influenza and those in previous years with seasonal influenza included only children considered to have died of influenza.

In this article, we present our experience with children admitted to hospital with pandemic H1N1 influenza. Our primary goal was to describe the demographic characteristics, clinical features and markers of severity of illness of these children. Our secondary goal was to identify risk factors for severe disease or poor outcome by comparing these children with those who had been admitted in previous years with seasonal influenza.

\section{Methods}

\section{Study design}

We reviewed the charts of all children admitted in 2009 to The Hospital for Sick Children, Toronto, Ontario, with laboratory-confirmed pandemic H1N1 influenza. This hospital serves as the pediatric referral centre for the Greater Toronto Area and the surrounding region. Every year, it admits about 40 children under 18 years old with influenza $A$, of whom 6 on average require intensive care.

We included children with pandemic H1N1 influenza confirmed by means of reverse transcription polymerase chain reaction using primers developed by the National Microbiology Laboratory, Winnipeg, Manitoba. ${ }^{8}$ We excluded children admitted more than 3 days before the onset of influenza-like symptoms, because the illness was deemed incidental to their admission.

We also reviewed the hospital charts of all children admitted during the previous 5 influenza seasons (2004/05-

From the Division of Infectious Diseases (O'Riordan, Barton, Read, Allen, Tran) and the Department of Paediatric Laboratory Medicine (Yau), The Hospital for Sick Children, University of Toronto, Toronto, Ont.; and the Department of Health Policy, Management and Evaluation (Barton, Allen), University of Toronto, Toronto, Ont. Sean O'Riordan and Michelle Barton are joint first authors.

CMAJ 2009. DOI:10.1503/cmaj.091724 
2008/09) who had seasonal influenza A detected by means of direct fluorescent antibody assay or viral culture. Similar exclusion criteria were applied.

The study was approved by the Research Ethics Board of The Hospital for Sick Children. The requirement for individual patient consent was waived for this retrospective cohort study.

\section{Variables of interest}

For the children with pandemic H1N1 influenza, we extracted the following data from their hospital charts: underlying medical conditions known to be risk factors for influenza-related complications, ${ }^{9}$ clinical features at presentation, influenzarelated complications, clinical course and outcome. For children admitted with seasonal influenza, we collected a more limited data set, comprising predisposing conditions and indicators of severity of illness.

We chose outcome measures that we felt could be reliably extracted in a retrospective chart review: mortality, requirement for intensive care or mechanical ventilation, length of stay in hospital and length of stay in the intensive care unit (ICU). Independent variables, selected a priori, were risk factors associated with severe influenza. ${ }^{9}$

We considered any prior diagnosis of asthma or reactive airways disease, or a history of wheeze necessitating inhaled medications to be indicative of asthma in the absence of a known diagnosis of another respiratory condition. Wheeze at presentation without a history of wheeze was not considered to be indicative of asthma. For questionable cases, consensus was sought among 3 reviewers (S.O., M.B. and D.T.).

To estimate the severity of asthma, we reviewed chart data on the level of use of asthma medications and the degree of asthma control. Severity was classified as mild (occasional use of inhaled medications and upper respiratory tract infections only), moderate (regular use of inhaled medications and good control) or severe (regular use of inhaled medications and poor control). Two children who used inhaled medications occasionally but who had a history of serious exacerbations were classified as having moderate rather than mild asthma. One of them had previously been admitted to an ICU because of asthma; the other had received oral steroid therapy at least twice previously because of exacerbations of wheeze necessitating a visit to the emergency department.

\section{Statistical analysis}

We performed comparisons between data from the group with pandemic H1N1 influenza and aggregated data from the group with seasonal influenza $\mathrm{A}$, and across all 5 seasons that preceded the pandemic H1N1 influenza season. We analyzed differences in normally distributed continuous variables between the comparator groups using Student's $t$ test or analysis of variance. We used nonparametric Mann-Whitney and Kruskal-Wallis methods for comparisons of the skewed length of stay and age data. The $\chi^{2}$ test or Fisher's exact test was used to compare categorical variables between groups and to test for heterogeneity among multiple proportions. We conducted univariable analysis to determine risk factors significantly associated with admission because of pandemic H1N1 influenza. Correction for multiple testing was carried out where specified in the text. We performed logistic regression to identify independent risk factors.

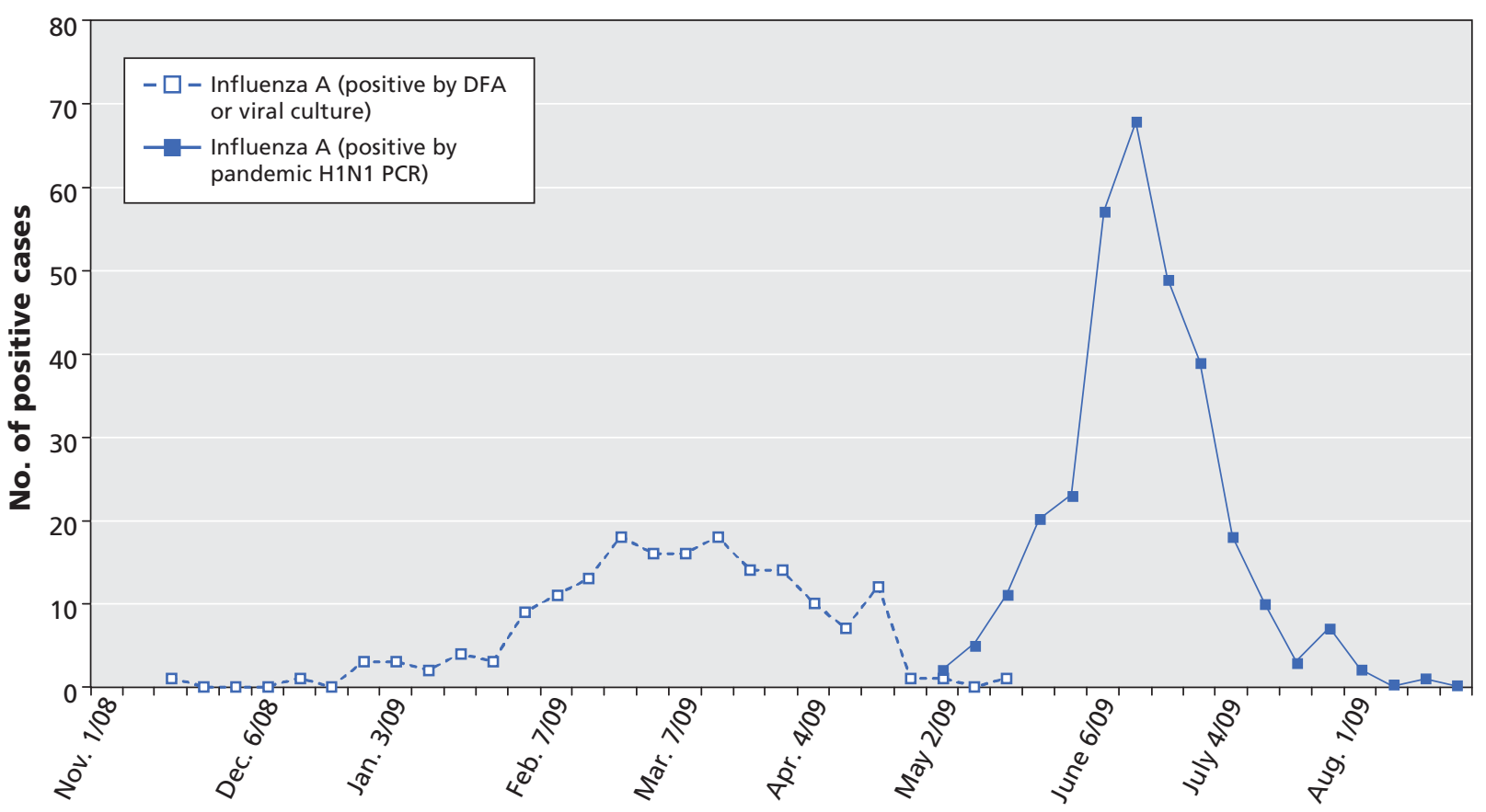

Figure 1: Epidemic curve of seasonal influenza A and pandemic H1N1 influenza at a large pediatric hospital in Toronto, Canada. Specimens originated from inpatients, outpatients and patients seen in the emergency department. Pandemic H1N1 polymerase chain reaction (PCR) testing was introduced on Apr. 28, 2009. Some cases of influenza A detected by means of direct fluorescent antibody assay (DFA) or viral culture before this date may have been pandemic H1N1 influenza. 


\section{Results}

We identified 61 children admitted with pandemic H1N1 influenza. We excluded 3 because the onset of symptoms occurred late in their admission. The first child was admitted on May 8, 2009, and the last on July 22, 2009. Figure 1 depicts the epidemic curve for the entire 2008/09 influenza season, including cases of pandemic H1N1 influenza.

A total of 200 children were admitted with seasonal influenza A during the previous 5 influenza seasons. We present aggregated data for these children because there were no significant differences across the seasons in terms of demographic characteristics, risk factors or outcomes.

\section{Demographic characteristics}

The children admitted with pandemic H1N1 influenza were significantly older than those admitted with seasonal influenza, with a significantly larger proportion over the age of 5 years (Table 1 and Figure 2). There were no differences in sex distribution.

\section{Clinical features of pandemic H1N1 influenza}

Forty-nine of the 58 children with pandemic H1N1 influenza presented with an influenza-like illness (fever and cough with or without additional signs or symptoms). Presenting symptoms in the remaining 9 children were fever and seizures (2), fever and diarrhea (2), fever alone ( 2 children with immunosuppression); lethargy without fever ( 1 child with cystic fibrosis); headache without fever (1 child who had received a renal transplant with a ventriculoperitoneal shunt); and fever and transient hematochezia (1). Including this last patient, 21 children $(36 \%)$ presented with gastrointestinal symptoms consisting of isolated vomiting (7) and vomiting with or without diarrhea (14). Four of these children experienced severe diarrhea; 2 had surgical gastrointestinal stomata, and 1 had presumed toxic shock syndrome.

Influenza-related encephalopathy was suspected in a previously healthy girl less than 5 years old who presented with status epilepticus and altered level of consciousness. Magnetic resonance imaging revealed nonspecific changes in the white matter, and electroencephalography showed focal slowing. Examination of cerebrospinal fluid yielded normal results; results of viral culture and polymerase chain reaction of cerebrospinal fluid were negative. Three other children presented with neurologic symptoms but had probable alternative explanations (see Appendix 1, available at www.cmaj .ca/cgi/content/full/cmaj.091724/DC1).

Seventeen children $(29 \%)$ had radiographic changes compatible with pneumonia. In a child with multiple medical problems and radiographic evidence of pneumonia, blood culture yielded Streptococcus pneumoniae.

\section{Severity of illness and outcomes}

The median duration of hospital stay was 4 days (interquartile range 2-7 days) for both the children with pandemic H1N1 influenza and those with seasonal influenza. We found no significant differences between the groups in the need for intensive care or mechanical ventilation $(7$ [12\%] and 19 [10\%] respectively, $p=0.57$ ). One child required extracorporeal membrane oxygenation. The trend toward longer duration of intensive care for children with pandemic H1N1 influenza did not reach statistical significance (Table 1). Two children with pandemic H1N1 influenza had prolonged stays in the ICU (30 and 61 days), which culminated in hospital stays of 69 and 65 days respectively. No deaths occurred among the children with pandemic H1N1 influenza, as compared with 1 death among those with seasonal influenza.

\section{Underlying medical conditions}

Underlying medical conditions known to be risk factors for severe influenza ${ }^{9}$ were present in $46(79 \%)$ of the children admitted with pandemic H1N1 influenza and 146 (73\%) of those admitted with seasonal influenza. Five of the 12 previously healthy children with pandemic H1N1 were less than 2 years old. Table 2 shows the distribution of selected risk factors for severe influenza. Children with pandemic H1N1 influenza were more likely to have asthma than children with seasonal influenza. Asthma remained a significant independent risk factor after we controlled for age by logistic regression $(p=0.001)$.

Among the children admitted with pandemic H1N1 influenza, the spectrum of asthma severity appeared broad (see Appendix 2, available at www.cmaj.ca/cgi/content/full /cmaj.091724/DC1) and did not seem to predict the need for
Table 1: Characteristics of 58 children admitted with pandemic H1N1 influenza in 2009 and 200 children admitted with seasonal influenza A during 2004-2009

\begin{tabular}{|lccc}
\hline Characteristic & $\begin{array}{c}\text { Pandemic H1N1 } \\
\text { influenza } \\
n=58\end{array}$ & $\begin{array}{c}\text { Seasonal } \\
\text { influenza A } \\
n=200\end{array}$ & $p$ value \\
\hline Sex, male, no. (\%) & $35(60)$ & $108(54)$ & 0.39 \\
\hline Age, yr, median (IQR) & $6.4(3.4-10.1)$ & $3.3(1.4-7.8)$ & $<0.001$ \\
\hline Age group, yr, no. (\%) & & & \\
\hline$<2$ & $8(14)$ & $74(37)$ & $<0.001$ \\
\hline $2-5$ & $13(22)$ & $54(27)$ & 0.48 \\
\hline$>5$ & $37(64)$ & $72(36)$ & $<0.001$ \\
\hline Admitted to intensive care unit & $12(21)$ & $28(14)$ & 0.21 \\
\hline No. (\%) of children & $5.8(3.2-7.3)$ & $3.1(1.4-8.7)$ & 0.57 \\
\hline Age, yr, median (IQR) & & & \\
\hline Required mechanical ventilation & $7(12)$ & $19(10)$ & 0.57 \\
\hline No. (\%) of all children & $7(58)$ & $19(68)$ & 0.72 \\
\hline No. (\%) of children admitted to ICU & & & \\
\hline Length of stay, d, median (IQR) & $4(2-7)$ & $4(2-7)$ & 0.59 \\
\hline In hospital & $3.5(2.5-17)$ & $2(1-5)$ & 0.06 \\
\hline In intensive care unit & & & \\
\hline
\end{tabular}

Note: $I Q R$ = interquartile range. 
intensive care, although numbers were small. Almost half of the children with pandemic H1N1 influenza who were admitted to the ICU had asthma (Table 2). Two children with clinical features suggestive of acute respiratory distress syndrome required mechanical ventilation, one of whom required extracorporeal membrane oxygenation. A further 2 were admitted to the ICU for management of their bronchospasm but did not require mechanical ventilation. Details of the 11 children with asthma admitted in previous influenza seasons are presented in Appendix 3 (available at www.cmaj.ca/cgi/content/full/cmaj.091724/DC1). No differences in clinical characteristics of asthma between the 2 groups of children were apparent, although small numbers precluded statistical analysis.

Data on height and weight were available for 35 of the children with pandemic H1N1 influenza. Two satisfied criteria for obesity, ${ }^{11}$ of whom 1 child, who also had asthma, required intensive care. Five of the 23 without height data had weights above the 97 th percentile. Two of these 5 children required intensive care: 1 child had asthma, and the other had seizures.

\section{Antimicrobial therapy}

Twelve (21\%) of the children with pandemic H1N1 influenza received antiviral therapy with oseltamivir. This comprised 8 children admitted to the ICU, 3 who had severe immunosuppression ( 2 of whom presented within 48 hours after symptom onset) and 1 who had presumed viral pneumonia and severe bronchospasm (with no previous history of asthma); this last patient was transferred to the ICU but was managed at a lower intensity. Three of the 4 children admitted to the ICU who did not receive antiviral therapy had shown marked clinical improvement by the time pandemic H1N1 influenza was confirmed. The fourth child (described in Appendix 1, at www.cmaj.ca/cgi/content/full/cmaj.091724/DC1) was less than 6 months of age, and influenza was not felt to be a sufficiently severe component of the illness for the benefits of treatment to outweigh the risks. The youngest child to receive oseltamivir in hospital was 2.5 years of age.

All but 2 of the children with pandemic H1N1 influenza received parenteral antibacterial therapy.

\section{Interpretation}

The presenting features of pandemic H1N1 influenza in the children in our series are similar to those described recently. ${ }^{6}$ The majority of the children in the initial series in the United States were between 10 and 18 years old; $;^{5}$ similarly, in a series in Chicago, the attack rate was higher in the group aged 5-14 years than in the group less than 5 years old. ${ }^{10}$ Data from Mexico on severe respiratory illness (regardless of definitive H1N1 diagnosis) showed a significant increase in the proportion affected among children aged 10-19 years, although not as dramatic as the increase among those aged $25-44$ years, and a corresponding decrease in the proportion affected among children less than 5 years old..$^{12}$ Against this apparent trend to increased age, the early Mexican epidemiologic studies found that children less than 2 years old were most

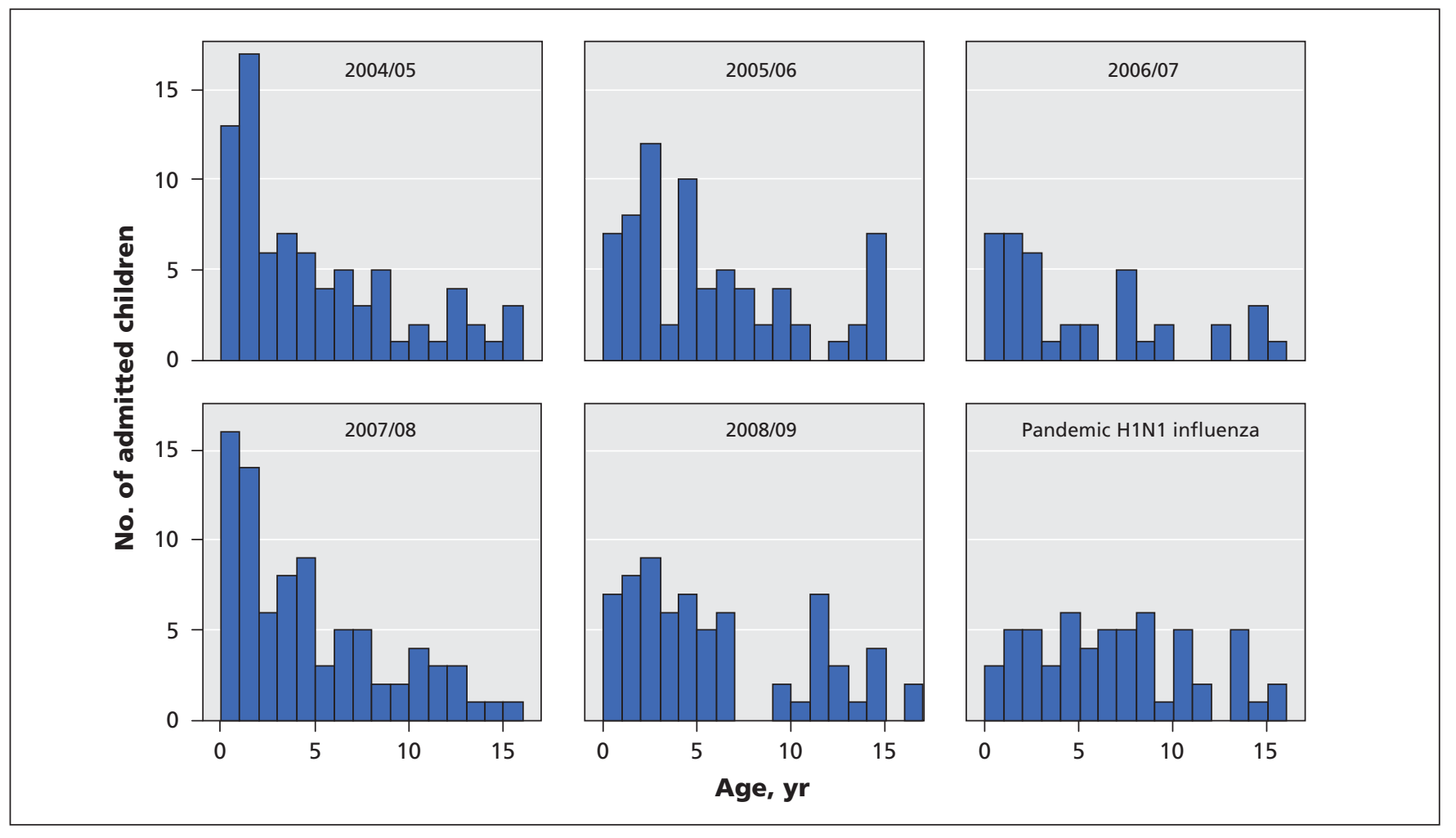

Figure 2: Age distribution of children admitted to hospital with seasonal influenza A (2004/05 to 2008/09) and pandemic H1N1 influenza (2009). 
affected, with attack rates falling progressively with increasing age. ${ }^{13}$ Both early case series of individuals admitted to hospital described children with a mean age more in keeping with our group with seasonal influenza.,4 The rate of hospital admission was highest among children less than 5 years old in both Chicago ${ }^{10}$ and New York. ${ }^{14}$

Detailed breakdown by age of our cohort reveals considerable year-to-year variability in the number of children less than 2 years old, with relatively low numbers in the group with pandemic H1N1 influenza (Figure 2). Although proportionately similar numbers were noted among children aged 2-5 years, the pattern was significantly different among children more than 5 years old; higher proportions of the children with pandemic H1N1 influenza were distributed in this older category (Table 1). Older children may have had some pre-existing or cross-reactive immunity to influenza strains circulating in previous seasons, which did not protect them against pandemic H1N1 influenza. ${ }^{15}$ However, community epidemiologic data are essential to assist in interpreting the observed difference in the ages of children admitted to hospital.

Our clinical impression of slightly more severe disease among admitted patients with pandemic H1N1 influenza was not borne out by rates or duration of ICU admission, or by length of hospital stay. Our impression remains that there is a small group of children who are more adversely affected by this virus. However, we have insufficient numbers to identify characteristics of this group. Obesity has been reported to be prevalent among patients with severe H1N1 influenza. ${ }^{6,16}$ In our cohort, all 3 children who were either obese (according to body mass index) or possibly obese (according to weight alone) and required intensive care had a high-risk medical condition. None of the children with pandemic H1N1 influenza in our study died, although 1 child required protracted extracorporeal membrane oxygenation therapy.

The most striking finding in our study was the high preva- lence of asthma among children admitted with pandemic H1N1 influenza compared with those admitted in previous influenza seasons. Asthma has been identified as a significant risk factor for pandemic $\mathrm{H} 1 \mathrm{~N} 1$ influenza requiring hospital admission, present in $21 \%-30 \%$ in the larger samples. ${ }^{10,14}$ However, there are few published pediatric data, particularly regarding the severity of asthma or the clinical course and outcomes of children with asthma. Comparisons between pandemic H1N1 influenza and seasonal influenza in children with asthma are lacking. Most prior data on seasonal influenza did not present asthma separately from other chronic lung diseases. Asthma or reactive airways disease was listed as part of the underlying condition in only 3 of 36 children who died of pandemic H1N1 influenza; all of these children also had neurologic impairment. ${ }^{7}$ Our experience suggests that asthma is a more significant risk factor for pandemic H1N1 influenza requiring hospital admission than for seasonal influenza and that children with mild asthma are also at risk. Of note, more than $50 \%$ of the children with asthma admitted to our hospital with pandemic H1N1 influenza presented with evidence of pneumonia, with or without bronchospasm.

Bias may have been introduced into our comparison of pandemic H1N1 influenza and seasonal influenza in previous years because of the possible influence of the pandemic publicity on admission criteria. We believe that this is unlikely, however, because a dedicated team of specialists offered emergency staff consistent, round-the-clock consultation on all potential admissions. Even if such a bias were to exist, it would apply across all underlying conditions and would not affect our conclusions regarding risk factors.

We cannot estimate the rate of admission to hospital because of pandemic H1N1 influenza without populationbased epidemiologic data. This is an area for imminent future research. Such studies should focus on age and underlying asthma in particular. There are limited data to support the

Table 2: Prevalence of underlying risk factors for severe influenza ${ }^{9}$ among children admitted with pandemic H1N1 influenza in 2009 and those admitted with seasonal influenza A during 2004-2009

\begin{tabular}{|c|c|c|c|c|c|c|}
\hline \multirow[b]{2}{*}{ Risk factor } & \multicolumn{3}{|c|}{ All children admitted to hospital } & \multicolumn{3}{|c|}{ Children admitted to intensive care unit } \\
\hline & $\begin{array}{c}\text { Pandemic H1N1 } \\
\text { influenza } \\
n=58\end{array}$ & $\begin{array}{c}\text { Seasonal } \\
\text { influenza A } \\
n=200\end{array}$ & $p$ value* & $\begin{array}{c}\text { Pandemic H1N1 } \\
\text { influenza } \\
n=12\end{array}$ & $\begin{array}{c}\text { Seasonal } \\
\text { influenza A } \\
n=28\end{array}$ & $p$ value* \\
\hline Asthma† & $13(22)$ & 11 (6) & $<0.001$ & $5(42)$ & $2(7)$ & 0.017 \\
\hline Chronic lung disease $¥$ & $2(3)$ & $13(6)$ & 0.53 & 0 & $5(18)$ & 0.30 \\
\hline Cardiac disease & $4(7)$ & $20(10)$ & 0.61 & $1(8)$ & $3(11)$ & 1.0 \\
\hline Hemoglobinopathy & $9(16)$ & $22(11)$ & 0.36 & $1(8)$ & 0 & 0.30 \\
\hline Immunodeficiency & $7(12)$ & $42(21)$ & 0.13 & 0 & $2(7)$ & 0.30 \\
\hline Neurologic impairment & $10(17)$ & $26(13)$ & 0.54 & $2(17)$ & $6(21)$ & 1.0 \\
\hline $\begin{array}{l}\text { Age }<2 \text { yr and no underlying } \\
\text { medical condition }\end{array}$ & $5(9)$ & 35 (18) & 0.15 & 1 (8) & $3(11)$ & 1.0 \\
\hline Other & 1 (2) & 12 (6) & 0.31 & $1 \quad(8)$ & $3(11)$ & 1.0 \\
\hline None & $7(12)$ & $19(10)$ & 0.74 & 1 (8) & $4(14)$ & 1.0 \\
\hline
\end{tabular}

* $\chi^{2}$ test (Fisher's exact test was used when cell sizes were small).

tLogistic regression analysis, with age as a continuous variable, showed an age-adjusted odds ratio for asthma of 4.38 (95\% confidence interval $1.81-10.60) ; p=0.001$. ‡Excluding asthma. 
efficacy of influenza vaccination in reducing asthma exacerbations, ${ }^{17}$ particularly in older children, ${ }^{18}$ although previous concerns about safety in asthma have been assuaged. Asthma has therefore been an indication for influenza vaccination for several years, but uptake is low. ${ }^{19}$ The current influenza season offers a good opportunity to improve the evidence base in this population and consequently change practice.

\section{Limitations}

This study is limited by its retrospective design, single-centre origin and lack of community epidemiologic data. Although this series remains the second largest published to date, and the largest from a single centre, the numbers of children admitted were small. This limits our ability to identify additional risk factors in subgroups of children and precludes multivariable analysis other than the exploration of the potential confounding effect of age on asthma as a risk factor. The indicators of influenza severity may have been influenced by factors unrelated to disease severity, such as availability of hospital or ICU beds. Nonetheless, we chose them as outcome measures because they have a major impact on resource planning, and because treatment-independent events, such as death and long-term sequelae from influenza infection, are too infrequent to be used as comparative outcome measures in samples of this size.

\section{Conclusion}

In our cohort, pandemic H1N1 influenza did not appear to cause more severe disease than seasonal influenza A. Asthma was a more significant risk factor for severe disease among children with pandemic H1N1 influenza than among those with seasonal influenza. Because the risk of severe disease appears to have no clear relation to the severity of asthma, even children with mild asthma should receive vaccination and be considered for pre-emptive antiviral therapy.

\section{This article has been peer reviewed.}

Competing interests: Upton Allen has previously received research funding from Hoffman-La Roche for work unrelated to this article. He participates on institutional, provincial and national committees related to pandemic influenza planning. No conflicts were declared by the other authors.

Contributors: Sean O'Riordan designed the study, gathered data and wrote the initial draft of the manuscript. Michelle Barton contributed to the design of the study, and the collection, analysis and interpretation of data. Yvonne Yau contributed the microbiological data. Stanley Read provided initial intellectual contribution that facilitated the study. Upton Allen conceived the study and contributed to the revision of the manuscript. Dat Tran conceived and designed the study, contributed to data collection and contributed to writing the manuscript. All of the authors critically revised the manuscript and approved the final version submitted for publication.
Acknowledgements: The authors acknowledge Kim Simpson and Suganya Lee for data management; Dr. Yan Li of the National Microbiology Laboratory, Winnipeg, for the development and supply of the primers for the pandemic H1N1 influenza reverse transcription polymerase chain reaction; the SickKids influenza management team; and Derek Stephens for statistical consultation.

Funding: No specific funding was received for this study. Dat Tran is supported by an Institute of Human Development, Child and Youth Health / SickKids Foundation New Investigator Grant (grant no. XG08-049R), a Canadian Institutes of Health Research Catalyst Grant (grant no. CAT86860) and a University of Toronto Dean's Fund Pilot Study grant.

\section{REFERENCES}

1. Iskander M, Booy R, Lambert S. The burden of influenza in children. Curr Opin Infect Dis 2007;20:259-63.

2. American Academy of Pediatrics. Influenza. In: Pickering LK, editor. 2009 red book: report of the Committee on Infectious Diseases. 28th ed. Elk Grove Village (IL): American Academy of Pediatrics; 2009. p. 400-12.

3. Hospitalized patients with novel influenza A (H1N1) virus infection - California, April-May, 2009. MMWR Morb Mortal Wkly Rep 2009;58:536-41.

4. Perez-Padilla R, de la Rosa-Zamboni D, de Leon SP, et al. Pneumonia and respiratory failure from swine-origin influenza A (H1N1) in Mexico. N Engl J Med 2009; 361:680-9.

5. Dawood FS, Jain S, Finelli L; Novel Swine-Origin Influenza A (H1N1) Virus Investigation Team. Emergence of a novel swine origin influenza A (H1N1) virus in humans. N Engl J Med 2009;360:2605-15.

6. Jain S, Kamimoto L, Bramley AM, et al. Hospitalized patients with 2009 H1N1 influenza in the United States April-June 2009. N Engl J Med 2009:361:1935-44.

7. Surveillance for pediatric deaths associated with 2009 pandemic influenza A (H1N1) virus infection - United States, April-August 2009. MMWR Morb Mortal Wkly Rep 2009;58:941-7.

8. Cutler J, Schleihauf E, Hatchette TF, et al. Investigation of the first cases of human-to-human infection with the new swine-origin influenza A (H1N1) virus in Canada. CMAJ 2009;181:159-63.

9. National Advisory Committee on Immunization. Statement on influenza vaccination for the 2008-2009 season. Can Commun Dis Rep 2008;34:ACS-3. Available: www.phac-aspc.gc.ca/publicat/ccdr-rmtc/08vol34/acs-3/index-eng.php (accessed 2009 Nov. 6).

10. 2009 pandemic influenza A (H1N1) virus infections - Chicago, Illinois, AprilJuly 2009. MMWR Morb Mortal Wkly Rep 2009;58:913-8.

11. Cole TJ, Bellizzi MC, Flegal KM, et al. Establishing a standard definition for child overweight and obesity worldwide: international survey. BMJ 2000;320:1240-3.

12. Chowell G, Bertozzi SM, Colchero MA, et al. Severe respiratory disease concurrent with the circulation of H1N1 influenza. N Engl J Med 2009;361:674-9.

13. Fraser C, Donnelly CA, Cauchemez S, et al. Pandemic potential of a strain of influenza A (H1N1): early findings. Science 2009;324:1557-61.

14. New York City Department of Health and Mental Hygiene. 2009 New York City Department of Health and Mental Hygiene health alert \#27: Pandemic (H1N1) 2009 influenza update, revised reporting requirements and testing procedures. New York (NY): The Department; 2009. Available: www.nyc.gov/html/doh /downloads/pdf/cd/2009/09md27.pdf. (accessed 2009 Nov. 6).

15. Serum cross-reactive antibody response to a novel influenza A (H1N1) virus after vaccination with seasonal influenza vaccine. MMWR Morb Mortal Wkly Rep 2009; 58:521-4.

16. Intensive-care patients with severe novel influenza A (H1N1) virus infection Michigan, June 2009. MMWR Morb Mortal Wkly Rep 2009;58:749-52.

17. Cates CJ, Jefferson TO, Rowe BH. Vaccines for preventing influenza in people with asthma [review]. Cochrane Database Syst Rev 2008;(2):CD000364.

18. Smits AJ, Hak E, Stalman WA, et al. Clinical effectiveness of conventional influenza vaccination in asthmatic children. Epidemiol Infect 2002;128:205-11.

19. Influenza vaccination coverage among children with asthma - United States, 2004-2005 influenza season. MMWR Morb Mortal Wkly Rep 2007;56:193-6.

Correspondence to: Dr. Dat Tran, Division of Infectious Diseases, The Hospital for Sick Children, 555 University Ave., Toronto ON M5G1X8; dat.tran@sickkids.ca 